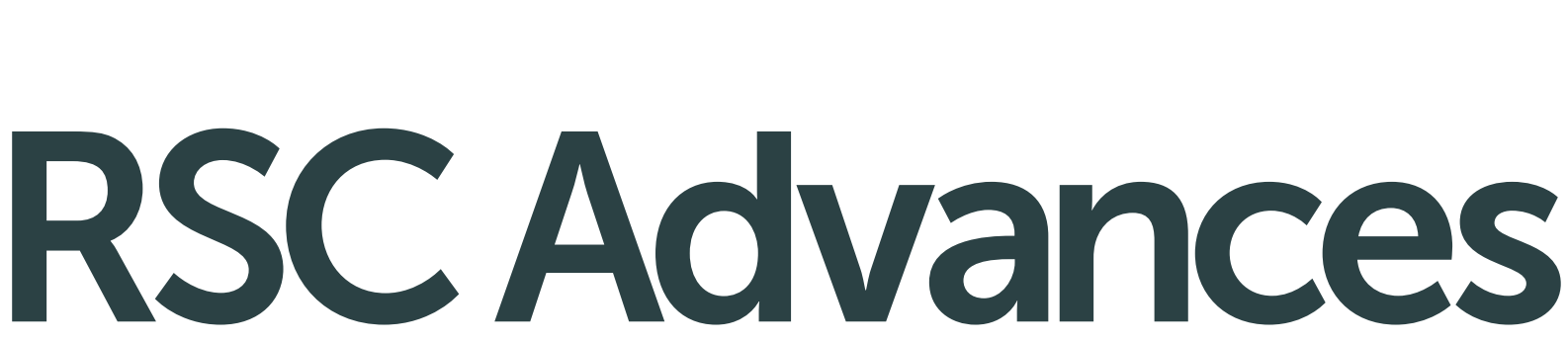

This article can be cited before page numbers have been issued, to do this please use: M. C. Righi, S. Loehlé, M. I. De Barros-Bouchet, S. Mambingo-Doumbe and J. Martin, RSC Adv., 2016, DOI:
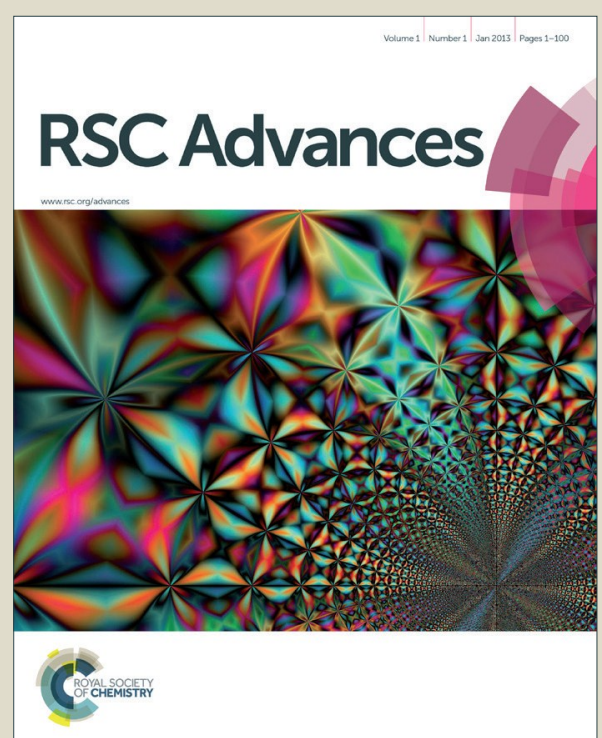

This is an Accepted Manuscript, which has been through the Royal Society of Chemistry peer review process and has been accepted for publication.

Accepted Manuscripts are published online shortly after acceptance, before technical editing, formatting and proof reading. Using this free service, authors can make their results available to the community, in citable form, before we publish the edited article. This Accepted Manuscript will be replaced by the edited, formatted and paginated article as soon as this is available.

You can find more information about Accepted Manuscripts in the Information for Authors.

Please note that technical editing may introduce minor changes to the text and/or graphics, which may alter content. The journal's standard Terms \& Conditions and the Ethical guidelines still apply. In no event shall the Royal Society of Chemistry be held responsible for any errors or omissions in this Accepted Manuscript or any consequences arising from the use of any information it contains. 


\title{
A comparative study on the functionality of S- and P-based lubricantit 1075455 additives by combined first principles and experimental analysis
}

\author{
M.C. Righi ${ }^{1,2^{*}}$, S. Loehlé ${ }^{3}$, M.I. De Barros Bouchet ${ }^{4}$, S. Mambingo-Doumbe ${ }^{3}$ and J. M. Martin ${ }^{4}$
}

1) Department of Physics, Informatics and Mathematics, University of Modena e Reggio Emilia

2) Instituto di Nanoscienze del CNR, centro $S 3$ di Modena

3) Total Research Center, Chemin du Canal BP 22, 69360 Solaize, France

4) LTDS, Ecole Centrale Lyon, Université de Lyon, 36 Avenue Guy de Collongue, 69134 Ecully, France

*Corresponding authors: mcrighi@unimore.it and maria-isabel.de-barros@ec-lyon.fr

\begin{abstract}
Sulfur and phosphorus are key elements for the functionality of lubricant additives used in extreme pressure applications, as synchronizers systems in cars. To understand their mechanism of action we combine first principles calculations and gas phase lubrication experiments. The surface spectroscopy analysis performed in situ after the tribological test indicates that iron sulfide (phosphide) is formed by rubbing steel-on-steel in the presence of organo-sulfur (-phosphorus) molecules. We, thus, study the effects of elemental sulfur and phosphorus on interfacial properties of iron by spin-polarized density functional theory calculations. The results show that both the elements are very effective in reducing the adhesion and shear strength of iron. Sulfur is predicted to be more effective than phosphorus, especially at high pressure. Gas phase lubrication experiments confirm these results, indicating that the friction coefficient of iron-suphide is lower than that of iron-phosphide and both $\mathrm{S}$ and $\mathrm{P}$ dramatically reduce the friction of steel-on-steel. These results indicate that the release of elemental sulfur and phosphorus may be the key mechanism to control the tribological properties of the metal interface and elucidate that the underling microscopic phenomenon is the metal passivation.
\end{abstract}

\section{Introduction}

The design of lubricants to decrease friction and wear in machine components is an important way to increase the energy efficiency of mechanical systems while taking into account restrictive environmental requirements and technological advances [1]. Lubricants are formulated products composed of base oil and a package of additives designed for specific performance needs. The additives can be classified as chemically active, i.e., designed to chemically interact with the surface and form protective layers, or chemically inert, i.e., with the function of improving the physical properties of the bulk materials. Most of lubricant additives have a non-polar part, usually consisting of an hydrocarbon chain, the functionality of which is to solubilize the molecule into the base oil and a functional polar group that reacts with the surface. Here we consider extreme-pressure (EP) and anti-wear (AW) additives where the key elements of the functional group are sulfur and phosphorus. 
EP, AW additives are typically used in synchronizer systems [2], which reduce the speed differemedecicle Online between the shaft and the idler gear [3]. The synchronizing system is a key component of manually operated vehicle transmissions, as fluent transmission operations have become increasingly important to drivers in the recent years. Gear oils must perform in conditions and applications that can vary drastically, therefore they are enriched by additives that protect seals, improve thermal, oxidative and viscosity stability, provide micropitting resistance, bearing corrosion protection, foam resistance, enhanced demulsibility and load-carrying capacity. Additives that work in severe conditions are EP and AW additives [4], their concentration is higher in gear oils, which are enclosed in gear boxes, than in engine oils [5].

S- and P-based compounds are largely used as EP and AW additives for gear oils [6-8]. EP additives typically adsorb onto the metal surface either by physical or chemical attraction $[9,10]$. Under severe tribological conditions, they react with the surfaces forming surface films that prevent the welding of opposing asperities and avoid scuffing that is destructive to sliding surfaces under high loads [11].

The action mechanism of the sulfur-based EP/AW additives was first proposed by Davey et al. [12] and then refined by Forbes et al. [13]: The sulfur compounds adsorb on an iron surface and under tribological conditions form inorganic iron sulfide films, which can reduce frictional wear and prevent seizure. The Fe-S formation under boundary lubrication conditions has been identified in different studies [14-16]. Recently, Li et al. considered three novel S-containing alkyl phenylboric esters [17, 18]. After the tribotest, the worn surface was investigated by X-ray absorption near edge structure spectroscopy (XANES) and Fourier transform infra-red (FT-IR) spectroscopy. The surface analysis confirmed the presence of iron sulfide.

The chemical structure of P-containing additives influences the nature and the tribological performances of the tribofilm as highlighted in [19-22], where phosphite and phosphate have been compared. Typically, organic phosphites function as friction-modifiers whereas phosphates as antiwear additives. Post mortem analysis of the boundary lubrication film formed on frictional surfaces showed that an iron phosphate compound is often obtained from phosphates [15, 22-24]. First, the organic phosphate molecule adsorbs on the iron substrate and then, iron phosphate is formed due to thermal/mechanical decomposition [26-31], but also due to hydrolysis [23, 25]. The formation of iron phosphate as a boundary lubrication film that prevents wear-out has been observed with steel ballon-disc tribotest followed by X-ray photoelectron spectroscopy (XPS) [33]. Philippon et al. have proposed a decomposition mechanism of a model EP additive, trimethyl-phosphite (TMPi), on nascent iron surface [34] that leads to the formation of iron phosphide film under tribological condition [35-37].

Such decomposition mechanism has been studied by first principle calculations and the reaction paths and energy barriers for $P$ release from the TMPi molecule have been provided [31]. Then, the functionality of elemental phosphorus in reducing friction of iron-based interfaces has been elucidated. It has, in fact, been shown that the work of separation and shear strength of iron interface dramatically decreases by increasing the phosphorus concentration at the interface [20]. These studies confirmed that first-principle calculations are able to provide an accurate description of the surface chemistry and interfacial properties, which is relevant for understanding the functionality of lubricant additives, since probing tribochemistry processes in real-time by experiments is still extremely challenging [38-42]. 
Here, we investigate the surface chemistry and its effects on interfacial properties of iron interfaredscle Online terminated either by sulfur or phosphorus elements and compare the results with experimental analysis. As mentioned above, the tribological performance of EP/AW additives containing $S$ and $P$ elements is linked to their release at the surface and to the formation of iron sulfide/phosphide tribofilms. In the present work, it is therefore assumed that additives are already decomposed under tribological conditions and only $\mathrm{S}$ and $\mathrm{P}$ elements are considered at the iron interface. Gas phase lubrication (GPL) experiments with in situ surface analysis are carried out in the presence of S- and Pcontaining molecules to analyze the macroscopic tribological behavior and identify the tribofilm chemistry. This study confirms that the combination of first principles calculations and experimental simulations offers one of the unique capabilities for investigating boundary lubricated interfaces, gaining insight into the friction reduction mechanism of EP/AW lubricant additives.

\section{Systems and methods}

We perform spin-polarized density functional theory (DFT) calculations [43], using the Perdew, Burke, Ernzerhof (PBE) parameterization for the exchange correlation functional [44]. The electronic wave functions are expanded in plane-waves with kinetic energy cut-off of 30 Ry (240 Ry for the charge density). The Brillouin zone of the $(1 \times 1)$ cell is sampled with a $8 \times 8$ Monckhorst Pack grid [45], equivalent samplings are realized for larger cells. To model an interface we use super cells with vertical axis $28 \AA ̊$ long containing two slabs of Fe three layers thick.

In order to construct the interface in a realistic way, we first study adatom adsorption on the $\mathrm{Fe}(110)$ surface and identify the most favorable adsorption site and coverage for both $\mathrm{P}$ and $\mathrm{S}$ elements. Then, we model the interfaces by self-mating the S- or P-terminated surfaces with the adatom arrangement identified as the most stable one.

The work of separation, which is defined as the energy per unit area required to separate two surfaces from contact, is calculated as $W_{\text {sep }}=\left(E_{\text {interf }}-E_{\text {surf1 }}-E_{\text {surf2 }}\right) / A$, where $E_{\text {interf }}$ is the total energy of the system containing the two surfaces in contact, $E_{\text {surf } 1,2}$ is the energy of the isolated surfaces, $A$ is the surface area. In the present work we consider interfaces composed of identical surfaces, therefore $E_{\text {surf } 1}=E_{\text {surf2 }}$.

Frictional forces arise during the displacement of one surface relative to another because the interaction energy between the two surfaces varies with their relative lateral position. This energy variation can be described as a potential energy surface (PES) with minima and maxima. The minima correspond to the relative positions where the surface adhere in the optimal way, for example, by establishing chemical bonds across the interface. The maxima of the PES correspond to relative positions where these bonds are stretched or eventually broken, therefore to displace one surface with respect to the other the energy to climb the PES maxima (or PES saddle points) should be provided to the system. The energy difference between the maxima and the minima is usually referred to as the PES corrugation. It corresponds to the maximum amount of energy that can be dissipated by frictional mechanisms, such as the excitation of phonons or the activation of chemical reactions that can modify the surface structure and composition. The PESes for the interfaces considered in the present study are constructed by calculating the adhesion energy for 7 different relative lateral positions and then interpolating. The calculations are performed both at zero and 10 
GPa applied loads. The frictional forces that arise when the slider is displaced along a s.mpmivetrivicle Online directions are then obtained by mathematical derivation of the PES profile along that directions [46]. The ideal interfacial shear strength is obtained by dividing the highest resistance force, $f$, along each considered direction by the contact area: $\tau=f / A$. $\tau$ represents the intrinsic resistance to sliding of a given interface. By comparing the shear strengths of different interfaces it is possible to understand which of them can be more easily set into sliding motion.

\section{Gas phase lubrication (GPL) with in situ surface analysis}

Tribochemical reactions of phosphite and polysulphide additives on steel surfaces are investigated using a specific Environmentally Controlled Analytical Tribometer (ECAT) dedicated to GPL $[16,20,47]$. This specific device is composed of a reciprocating pin-on-flat tribometer with a movable pin being connected to a brushless motor located in a Ultra High Vacuum (UHV) chamber. The temperature of the friction pair can be controlled from $-100^{\circ} \mathrm{C}$ to $600^{\circ} \mathrm{C}$ thanks to the flat holder equipped with both a nitrogen cooling and a heating system. The normal and tangential forces can be accurately measured thanks to optical sensors. With this GPL approach, the lubricant additives can be introduced under gas phase inside the tribological chamber by using 3 micro-leaks valves and the partial pressure can be continuously varied from $10^{-9} \mathrm{hPa}$ to $2000 \mathrm{hPa}$, (twice the atmospheric pressure), thanks to a membrane vacuum gauge. The tribometer is computer-controlled and the normal load is automatically re-adjusted when the gas pressure inside the tribometry chamber has changed. The main advantage of the ECAT device is the possibility to perform in situ XPS and Auger surface analyses on the rubbed surfaces at the end of the friction experiments without any exterior contamination. Recently, GPL approach has established clear differences between the tribochemistry of organophosphates and organophosphites molecules on the steel surface and evidenced the relationship with the tribological performances [31].

Here, GPL experiments are performed for two different kinds of molecules, a phosphorouscontaining additive dimethylphosphite (DMPi) and a sulfur-containing trisulfide additive (TPS44) (Figure 1). DMPi has a purity of $99+\%$ (> 99\%) and is provided by the Aldrich Chemical Co. The DMPi compound presents two chemical equilibrium states, a phosphite form in which the $\mathrm{P}$ atom is trivalent and a phosphonate form in which the $\mathrm{P}$ atom is pentavalent. The trisulfides (TPS44) is an industrial additive supplied by TOTAL Company, it contains some impurities, in particular oxygen. These molecules can be evaporated and introduced into the vacuum chamber until a limited pressure at room temperature, corresponding to the saturating vapor pressure (SVP), is reached because of their heavy molecular weight, specially the TPS44 compound (Figure 1). They are further purified by freeze pumping thaw cycles before being introduced into the tribometry chamber. Friction materials, pin and flat, used are made of AISI 52100 steel (composition $96.9 \mathrm{Fe}-1.04 \mathrm{C}-1.45$ $\mathrm{Cr}-0.35 \mathrm{Mn}-0.27 \mathrm{Si}$ in wt\%) and both the surfaces are polished with a 1 um diamond paste solution in order to obtain a surface roughness of about $20-25 \mathrm{~nm}$ in Ra, then cleaned with a n-heptane ultrasonic bath. The use of steel is preferred to pure iron crystal because the hardness of pure iron is too low to reach the required contact pressure. The pin has a hemispherical radius of $8 \mathrm{~mm}$. In order to match as close as possible the modeled conditions in the computational study, the $3-4 \mathrm{~nm}$ thick native oxide/hydroxide layer present at the top surface of the steel samples is removed using an $\mathrm{Ar}$ ion gun VG EXO5 (ions of $3 \mathrm{keV}$ ) to obtain a metallic iron surface. Before starting the GPL experiments, the etched sample is first analyzed by XPS to check its purity in iron, e.g. in the absence of any adventitious carbon and oxides layers. These GPL experiments are run for 600 cycles at room 
temperature with a normal applied load of $3.5 \mathrm{~N}$ corresponding to a maximum Hertzian colittacticle Online pressure of about $0.52 \mathrm{GPa}$ and a Hertz diameter of $114 \mu \mathrm{m}$. The track length is adjusted to about 2 $\mathrm{mm}$ and the sliding speed of the reciprocating motion is set at about $0.5 \mathrm{~mm} . \mathrm{s}^{-1}$. All the tests are repeated three times to check their reproducibility.

After GPL experiments, the rubbed steel samples are directly introduced into the analysis chamber without any cleaning step or air exposure. Auger Electron Spectroscopy (AES) analysis is carried out both inside and outside the wear scar thanks to an electron gun FEG1000 with a small spot size of 1 $\mu \mathrm{m}$. The AES electrons emitted by the surface during the analysis are detected using a VG 220i spectrometer. AES analysis presents both a high spatial resolution $(1 \mu \mathrm{m})$ and a clear identification of the oxidation state for $\mathrm{S}$ and $\mathrm{P}$ chemical elements.

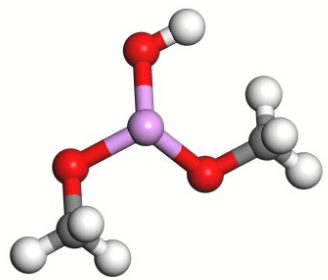

a)

Figure 1: DMPi molecule in the two states of a) dimethyl-hydrogen phosphite and b) dimethyl-phosphonate. c) Tertiary polysulfides (TPS44) molecule. The adopted color scheme is: $\mathrm{P}$ in purple, $\mathrm{O}$ in red, $\mathrm{S}$ in yellow, $\mathrm{C}$ in grey and $\mathrm{H}$ in white. The molecular weight of DMPi (TPS44) is $110 \mathrm{~g} / \mathrm{mol}(210 \mathrm{~g} / \mathrm{mol})$, and the SVP at room temperature is $30 \mathrm{hPa}(3 \mathrm{hPa})$.

\section{Results and discussion}

1. $A b$ initio study of $\mathrm{P}$ and $\mathrm{S}$ adsorption on $\mathrm{Fe}(110)$

To identify the most stable adsorption site for S and P atoms at the Fe(110) surface, we consider the (2x2) cell and position one adatom in different high-symmetry sites. A structural relaxation is performed by keeping fixed the lateral $(x, y)$ coordinates of the ad-atom and the bottom layer of the slab. The energy comparison reveales that the adatom stability increases with its coordination on the surface and the most favorable adsorption site for both S and P is the long-bridge (LB) site, where the atom can bind with four Fe atoms on the surface. This result is in agreement with previous DFT calculation of $S$ adsorption [48], while we are not aware of any previous study of $P$ adsorption on Fe(110). The adsorption distance between the adatom and the Fe surface atoms is $1.53 \AA$ and $1.51 \AA$ for $\mathrm{S}$ and $\mathrm{P}$ elements, respectively.

Different coverages of phosphorus and sulfur are simulated by varying the number of adatoms adsorbed at LB sites in the $(2 \times 2)$ cell. The considered configurations are reported in table 1 . We find that the most favorable coverage is 0.25 monolayer $(\mathrm{ML})$, which corresponds to one ad-atom per $(2 \times 2)$ cell. This result is in agreement with the LEED experimental observation of a $(2 \times 2)$ reconstruction formed on the Fe(110) surface upon S adsorption [49]. As can be seen in table 1, the adsorption energy increases with the adatom concentration and the energy cost to increase the coverage is higher in the case of $\mathrm{S}$ atoms than $\mathrm{P}$ atoms. 
Table 1 : Increase of adsorption energy with coverage (the percentage increase is calculated with respect to the imostcle Online stable configuration) corresponding to $1 / 4 \mathrm{ML}$ both for $\mathbf{S}$ and $\mathbf{P}$ adsorption. The adatom configurations corresponding to $\mathrm{A} 07545 \mathrm{~B}$ the considered coverages are reported in a top-view representation.

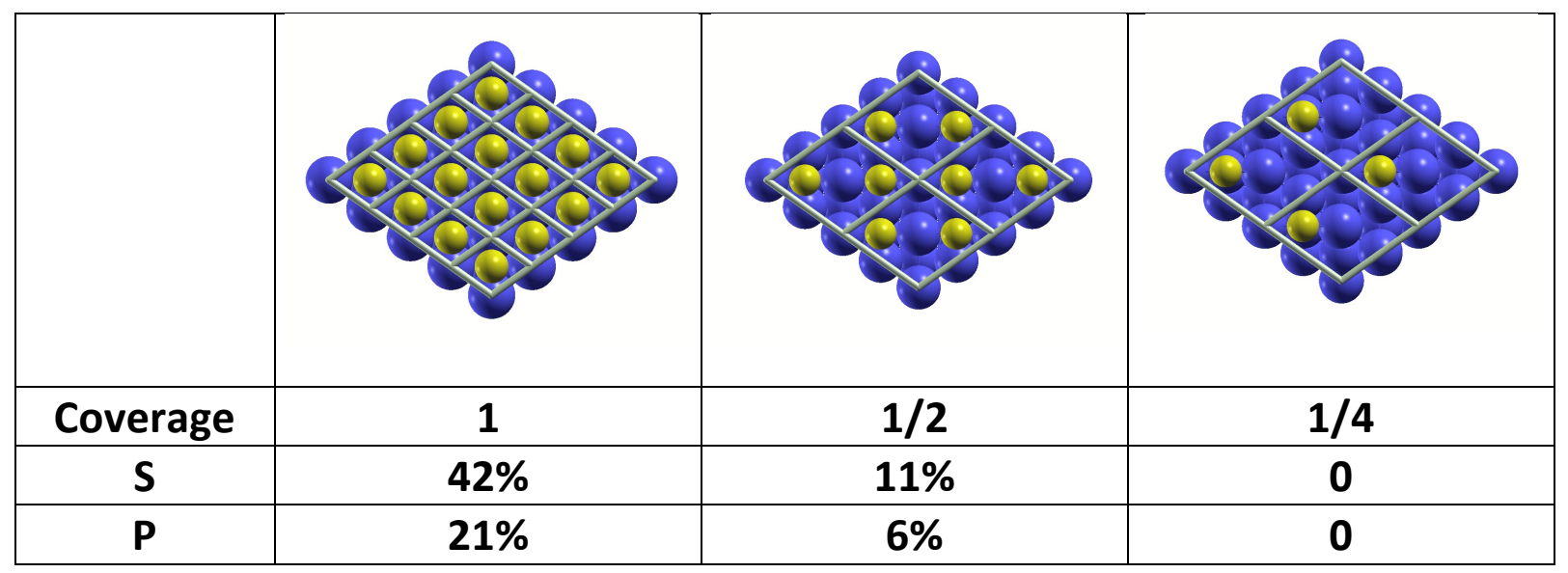

2) Interfacial adhesion

We construct S- and P-terminated interfaces by self-making $(2 \times 2)$ surfaces with the most favorable coverage, i.e, $1 / 4 \mathrm{ML}$. We study the variation of the adhesion energy of clean, P-passivated and S-passivated iron interfaces as a function of the separation between the two surfaces in contact. The calculations are performed by fixing the bottom layers of the two mating slabs, and allowing all the other system degrees of freedom to relax. The two surfaces are initially positioned at very close distance. Then, the system is allowed to relax until the equilibrium distance between the surfaces is reached. Table 2 represents the work of adhesion for clean and passivated iron interfaces in the most favorable relative lateral position. It is clear that both phosphorus and sulfur decrease the work of separation with respect to the clean interface, and $\mathrm{S}$ is five times more efficient in reducing the work of adhesion than $\mathrm{P}$. The separation between the metal surfaces increases in the presence of $S$ and $\mathrm{P}$, which is a favorable situation especially at high $\mathrm{S}$ and $\mathrm{P}$ coverages.

Table 2 : The work of separation, $W_{\text {sep, }}$ and equilibrium surface separation, $\Delta z$, calculated for clean and passivated iron interfaces.

\begin{tabular}{|c|c|c|c|}
\hline$\theta$ & 0 & $1 / 4 \mathrm{ML} \mathrm{P}$ & $1 / 4 \mathrm{ML} \mathrm{S}$ \\
\hline $\mathrm{W}_{\text {sep }}\left(\mathrm{J} / \mathrm{m}^{2}\right)$ & 4.8 & 2.5 & 0.5 \\
\hline$\Delta z(\AA)$ & 2.4 & 3.2 & 3.4 \\
\hline
\end{tabular}

Figure 2 reports the adhesion energy per $1 \times 1$ cell as a function of the interfacial separation. Negative values of the adhesion energy indicate an attractive interaction between the surfaces, while positive values indicate repulsion between the surfaces. Each dot in the picture is the result of a first principles calculation. Aside of each dot is reported the value of the pressure in GPa calculated from the residual forces on the fixed layers. Negative values indicate that a tensile stress should be applied to keep the surfaces at the corresponding distance, while positive pressures indicate that a compressive stress should be applied to reach the corresponding short distances. 


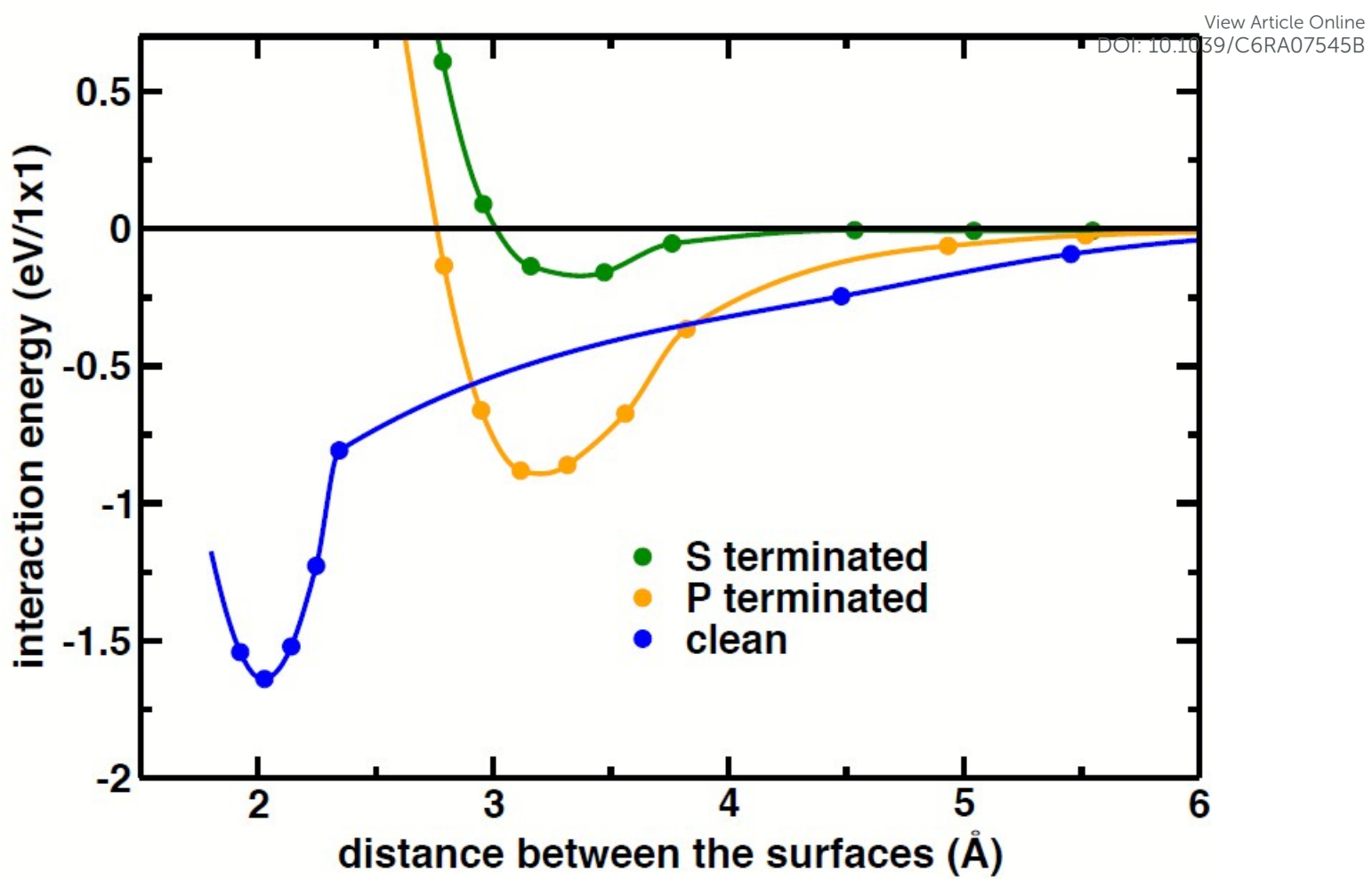

Figure 2: Adhesion energy as a function of the surface separation. The load (tensile or compressive) necessary to bring the surfaces at the considered distances is reported in GPa.

The minimum of the blue curve, which corresponds to the clean interface, is much deeper than the minima of the red and the black curves, representing respectively $\mathrm{P}$ - and S-passivated interfaces. This clearly indicates that the chemical adhesion of iron is highly reduced by the presence of passivating species. Large tensile stresses (up to $51 \mathrm{GPa}$ ) should be applied to increase the separation between the clean iron surfaces from the equilibrium value, which corresponds to the Fe-Fe bond length. Finally, it can be observed that the interaction between the clean surfaces is attractive for a wide range of distances and it vanishes only when the surfaces are brought at about $6 \AA$ separation. The comparison between the curves corresponding to S- and P-passivation, reveals a lower adhesion in the case of S-passivation (the minimum of the black cure is much lower than that of the red curve). In addition, the interaction between the S-passivated surfaces is attractive for a much narrower range of distances (from $3 \AA$ to $4.5 \AA$ ) than that of the P-passivated ones (from $2.75 \AA$ to $6 \AA$ ) and lower tensile stresses should be applied to increase the separation between S-passivated than P-passivated surfaces.

3) Shear strength

A bidimensional representation of the PESes obtained for $\mathrm{S}$ and P-passivated sliding interfaces is shown in Fig. 3a, a common energy scale is used, where the minima are colored in blue. The ball-andstick inset offers a top view representation of the substrate surface. An identical surface shoud be imagined sliding on it. The labeled white dots indicate the relative positions considered in the calculation of the PES. The PES corrugation of the P-passivated interfaces is much higher than that of the S-passivated ones, consistently with the higher adhesion calculated for the P-passivated interface . In Fig. $3 \mathrm{~b}$ the PES profiles aling the three symmetry directions indicated by arrows in panel a) are 
displayed. The red (black) color is used for P (S) passivation (Figure $3 \mathrm{~b}$ )). It is noticed that in spitejeof fidcle Online more pronounced load-induced increase, the energy barriers that should be overcame to slide $\mathrm{S}$ passivated surfaces are lower than those of P-passivated surfaces. This produces lower frictional forces, as it can be seen in table 3 where the shear strengths are reported for the three considered directions.

a)

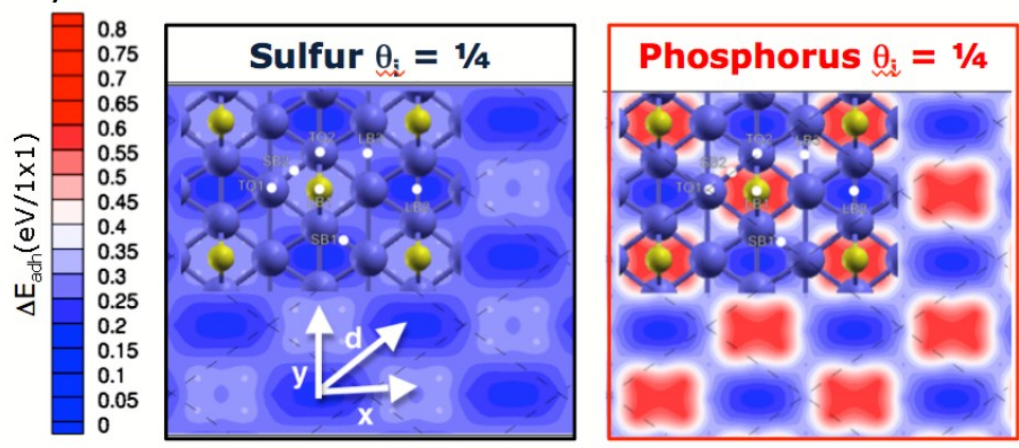

b)
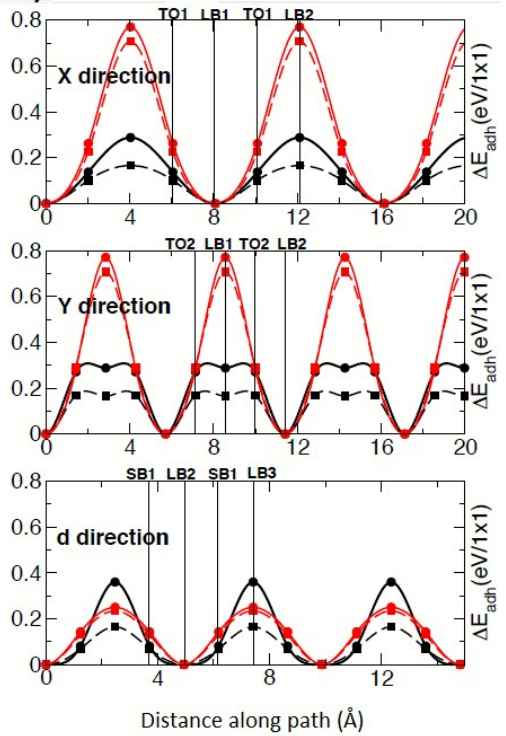

Figure 3 : panel a) Bidimensional representation of the PES obtained for S- and P- passivated interfaces at zero load, panel b) PES profiles along the three symmetry directions indicated with arrows in Fig. 3a. The red (black) color is used for the P- (S-)passivated interfaces, a continuous (dashed) line is used for $10 \mathrm{GPa}$ (zero) applied load.

If we consider the " $d$ " direction, which corresponds to the minimum energy path that connects the PES minima passing through the saddle points, we can notice that the resistance to sliding of a clean iron interface at zero applied pressure is of $4 \mathrm{GPa}$. A $60 \%$ reduction is obtained by including interfacial phosphorus in a small concentration of $0.25 \mathrm{ML}$. This reduction reaches the $70 \%$ if sulfur is used instead of phosphorus (third line of Tab. 3).

Table 3 : Ideal shear strengths along high symmetry directions for clean and passivated interfaces

\begin{tabular}{|l|l|l|l|}
\hline & Clean interface & P coverage $1 / 4 \mathrm{ML}$ & S coverage $1 / 4 \mathrm{ML}$ \\
\hline$\tau_{x}(\mathrm{GPa})$ & 32.5 & 8.9 & 1.9 \\
\hline$\tau_{y}(\mathrm{GPa})$ & 46.0 & 11.0 & 2.5 \\
\hline$\tau_{d}(\mathrm{GPa})$ & 10.2 & 4.1 & 3.0 \\
\hline
\end{tabular}

The surface passivation decreases the Fe-Fe interaction at the interface, decreasing friction and adhesion, especially at high coverage. Our calculations reveal that $\mathrm{P}$ and $\mathrm{S}$ are good passivating species as they create electrostatic and Pauli repulsion at short distances. The mechanism of surface passivation to reduce friction is effective also in other materials like diamond [50-52]. 
In the following, GPL friction experiments as well as in situ surface analysis have been performed todcle Online study experimentally the efficiency of passivated iron interfaces obtained by P-and S-based additives in lowering macroscopic friction. Results are then compared to simulations.

\section{4) Experimental results}

The evolution of the friction coefficients obtained with GPL in the presence of $1 \mathrm{hPa}$ of DMPi and $1 \mathrm{hPa}$ of TPS44 are shown in figure $4 \mathrm{a}$ ). It clearly appears that the trisulfide TPS44 molecule reduces friction more efficiently than the DMPi molecule at the same gas pressure. In fact, the friction coefficient reaches a steady-state value of about 0.18 for TPS44 compared to 0.30 for DMPi. Additionally, the friction coefficient obtained with etched steel surfaces under UHV is reported as reference. Both DMPi and TPS44 molecules reduce friction drastically in comparison with UHV. Moreover, these two compounds have good anti-wear properties because the width of the wear scars formed on the steel flats (Figure 4b)) almost corresponds to the calculated initial Hertzian diameter, i.e., $114 \mu \mathrm{m}$. The different frictional behaviors of the phosphorous and sulfur-containing moieties can be related to the chemical nature of the formed tribofilm.

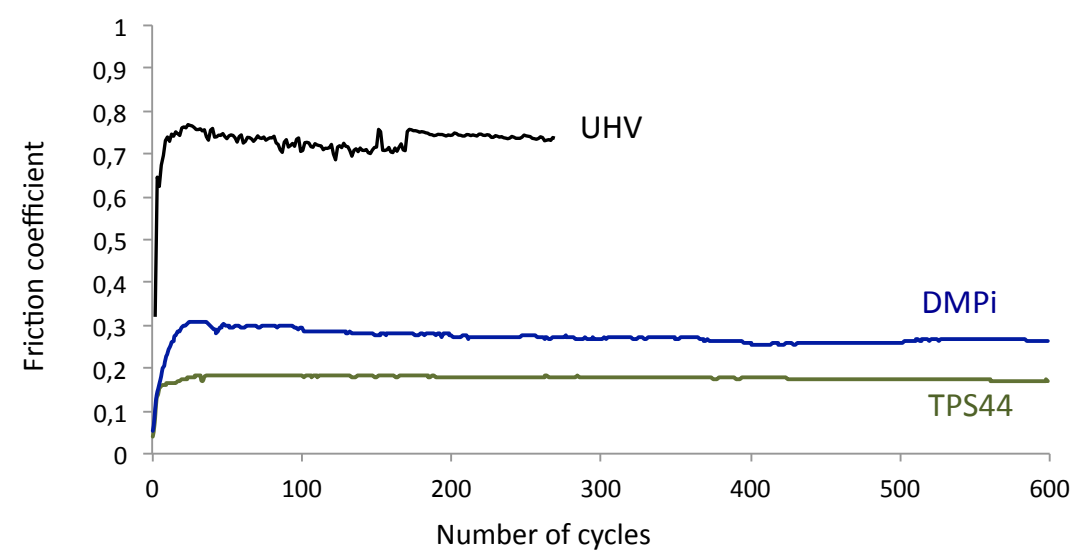

a)

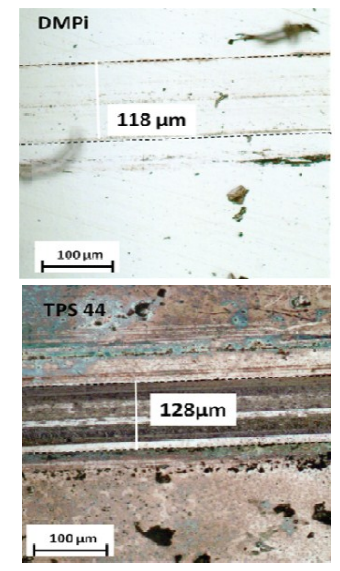

b)

Figure $4:$ a) Evolution of the friction coefficient obtained under GPL in the presence of $1 \mathrm{hPa}$ DMPi and $1 \mathrm{hPa}$ TPS44 at room temperature. The friction coefficient obtained under UHV is reported for comparison. b) Wear scars formed on the steel flats during GPL experiments.

The combination of GPL experiments with in situ surface analysis allows an accurate investigation of the chemical nature of the tribofilm with a clear assignment of the chemical state of the different elements. Figure 5 shows in situ AES spectra recorded inside and outside the DMPi- and TPS44derived tribofilms at the end of the GPL experiments. For DMPi compound, the tribofilm shows the presence of phosphide peaking at about $120 \mathrm{eV}$ confirming the decomposition of DMPi molecule under shearing with the release of $\mathrm{P}$ element to form P-Fe bonds. The phosphate chemical bond would show an AES peak at $110 \mathrm{eV}$, which is practically not observed here on the spectrum. By comparing the AES spectra recorded inside and outside the tribofilm, it clearly appears that $\mathrm{Fe}_{\mathrm{x}} \mathrm{P}$ iron phosphide generation is widely promoted under tribological conditions, in agreement with previous 
studies [53]. As shown by Figure 5, outside the tribofilm only the adsorption of the DMPi complourndidcle Online can be detected with a small contribution at about $110 \mathrm{eV}$. Regarding the trisulfide TPS44 compound, the formation of iron sulfide inside the tribofilm is clearly evidenced by the strong $S$ LMM peak at about $152 \mathrm{eV}$ confirming the decomposition of TPS44 under shear and the release of S element to form S-Fe bonds. Outside the TPS44-derived tribofilm, only a weak adsorption of sulfur as sulfide can be observed. The stronger intensity of the iron sulfide contribution in comparison with iron phosphide suggests a higher reactivity of the TPS44 compound on the iron surface and/or a higher stability of the S-Fe bond in comparison with P-Fe one. Surprisingly, we can also observe the presence of oxygen on the spectrum recorded on the TPS44-derived tribofilm although the molecule of TPS44 does not contain any oxygen. However, the amount of oxygen remains quite low and may be due to the presence of impurities in the TPS44 industrial products. Indeed, the production of organic polysulfides additives involves different oxygenated products [54, 55].

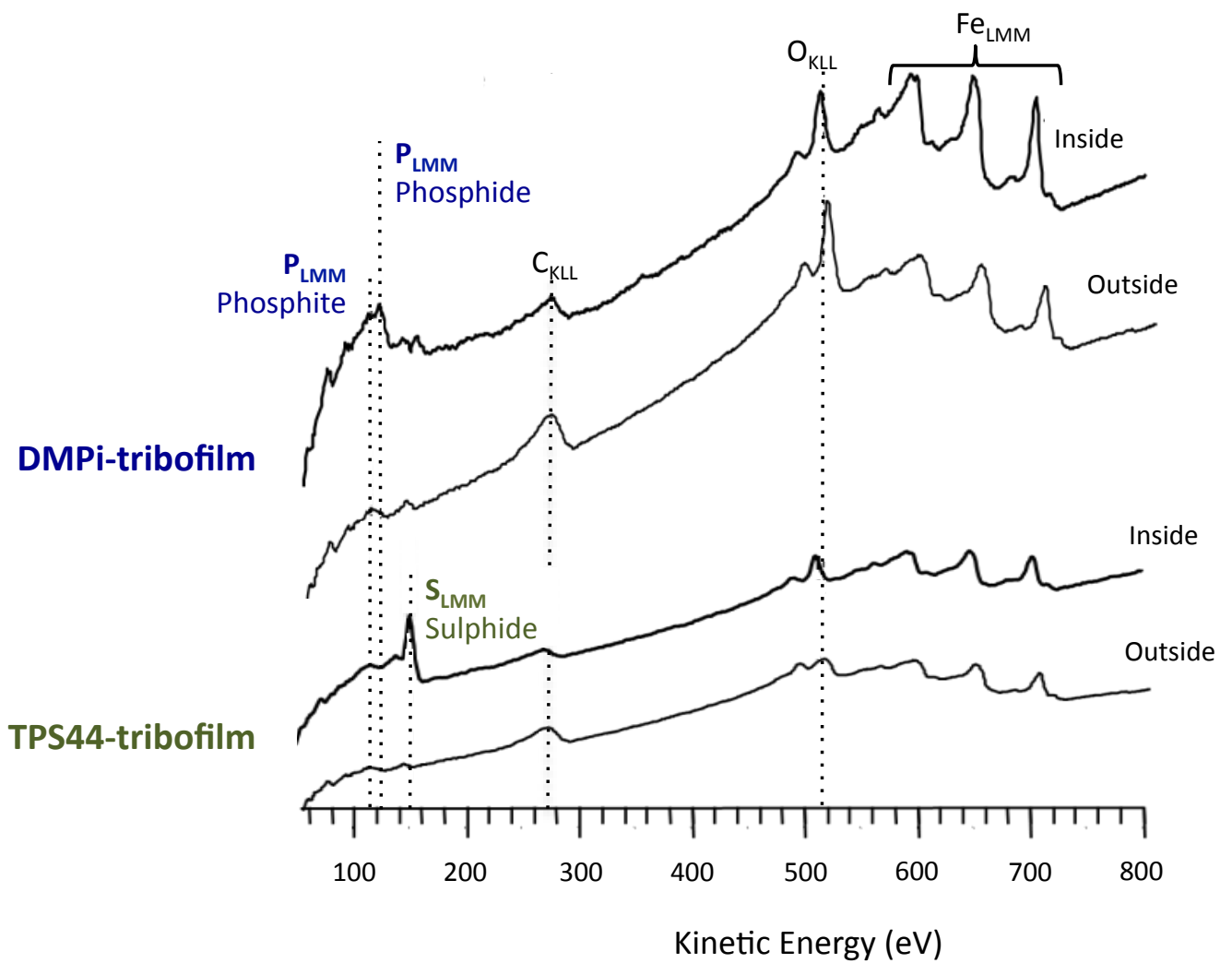

Figure 5: In situ AES spectra recorded inside and outside the DMPi-derived tribofilm and TPS44-derived tribofilm after GPL experiments.

The friction reduction property is correlated with the formation of iron phosphide and iron sulphide in the tribofilm. These experimental results are in agreement with previous experimental observations where it has been reported that the lower shear strength of sulfur-containing tribofilms compared to phosphorus-containing tribofilms is the reason for lower friction coefficient observed [56]. This is confirmed by our first principle calculations results, which reveal that phosphorus and sulfur are very efficient in reducing the adhesion and shear strength of iron-on-iron interface. 
Calculations and experiments are in agreement to show that $S$ is more effective than $P$ in redkicinificle Online friction.

\section{Conclusion}

We perform a firstprinciples comparative study on the effects of sulfur and phosphorus on interfacial properties of iron. We show that the metal passivation by these elements has a high impact on its tribological properties: a surface coverage of $0.25 \mathrm{ML}$, which we identify as the most energetically favored for both the species, is able to reduce the adhesion between iron surfaces very effectively, in particular sulfur. A detailed analysis of the surface interaction as a function of distance reveals, in fact, that the attraction between P-passivated surfaces is larger than between the S-passivated ones. We construct the potential energy surface (PES) for the sliding interface and derive the ideal interfacial shear strength. The results show that the intrinsic resistance to sliding of a clean iron interface can be reduced by $60 \%$ by including $0.25 \mathrm{ML}$ interfacial phosphorus and this reduction reaches $70 \%$ in the case of interfacial sulfur. These numerical results predict that sulfur is more efficient in reducing friction of iron-based surfaces than phosphorous.

Using a dedicated UHV tribometer coupled with in situ Auger spectroscopy, we run friction experiments on steel samples in the presence of partial pressures of dimethyl phosphite (DMPi) and an organic polysulfide (TPS 44). In situ AES analysis inside and outside area scars clearly show the formation of iron phosphide with DMPi and iron sulfide with TPS 44. The absence of formation of organic sulfates and phosphates compounds is clearly evidenced by GPL approach with in situ AES. Friction results show that TPS 44 (iron sulfide) is more efficient than DMPi (iron phosphide) in reducing friction of steel contact, in agreement with the results of firstprinciples calculations.

Overall, our study indicates that the approach that combines firstprinciples calculations and gas phase lubrication is very efficient to study tribochemistry mechanisms of lubricant additives.

\section{References}

[1] Spikes H, Tribology Letters 60:5 (2015)

[2] Wanli X, Wai Z, Bin S, Ximeng X, Wear 328-329 (2015) 475-479

[3] Bouffet A, SAE Paper 01-2022 (2004)

[4] Vinci J, Lubrizol Corporation US20060276352, (2006)

[5] Zakarian J, Haire M, Chevron Texaco Corporation, US20060252657, (2006)

[6] Matsui S, Komatsubara H, Nippon Oil Corporation, US20070287643, (2007)

[7] Craig T, Thomas T, Lubrizol Corporation, WO2007111741, (2007)

[8] Furlong O, Gao F, Tribology International 40, (2008) 699 
[9] Tang Z, Li S, Current Opinion in Solid State and Materials Science 18, (2014) 119

[10] Spikes H, Lubrication Science 20, (2008) 103

[11] Van Rensselar J, Power Transmission Engineering, April 2003

[12] Davey W, et al., Wear, 1, (1958) 291

[13] Forbes E. S and Reid A.J.D, ASLE Trans. 16 (1973) 50

[14] Coy R.C and Quinn T.F.J, ASLE Trans., 18 (1975) 163

[15] Mori S, Hori K, Tamai Y, Junkatsu 27, (1982) 505

[16] J. Tannous, Barros M.I. de Bouchet, Th. Le Mogne, P. Charles, J.M. Martin, Tribology-Materials, Surfaces and Interfaces, 1, (2007) 98

[17] Li Z, Li Y, Zhang Y, Ren T, Zhao Y, RSC Advances 4, (2014) 25118

[18] Li J, Fan B, Ren T, Zhao Y, Tribology International 88, (2015) 1

[19] A. Riga, J. Cahoon and W. R. Pistillo: Tribol. Lett., 9, (2000) 219

[20] M. I. De Barros-Bouchet, M. C. Righi, D. Philippon, S. Mambingo-Doumbe, T. Le-Mogne, J. M. Martin and A. Bouffet, RSC Adv., 5, (2015) 49270

[21] F. Gao, P. V. Kotvis, D. Stacchiola and W. T. Tysoe, Tribology Letter 18 (2005) 377-384.

[22] F. Gao, O. Furlong, P. V. Kotvis and W. T. Tysoe, Langmuir 20 (2004) 7557-7568.

[23] Beek O, Givens J.W, Williams E.C, Proc. Roy. Soc. A-177, (1940) 103

[24] Godfrey D, ASLE Trans., 8, (1965) 1

[25] Barcroft F.T and Daniel S.G, Trans. ASME, J. Basic Eng., 87D, (1965) 761

[26] Yamamoto Y et al., Wear 50, (1978) 343

[27] Sung D, Gellman A. J, Tribology Letters, 13, (2002) 1

[28] Holbert A. W, Batteas J. D, Wong-Foy A, Rufael T. S, Friend C. M, Surface Science 401 (1998) 1437

[29] Ren D, Zhou G, Gellman A. J, Surface Science 61, (2001) 475

[30] Ren D, Gellman A. J, Tribology International 34, (2001) 353

[31] Righi M.C, Loehlé S, De Barros-Bouchet M. I, Philippon D, Martin J.M, RSC Advances, 52015 101162

[32] Rossi A, Piras F. M, Kim D, Gellman A. J, Spencer N. D, Tribology Letters 23, (2006) 197

[33] Mistry K K, Morina A, Erdemir A, Neville A, Tribology Transactions 56, (2013) 4 
[34] Philippon D et al., Tribology International 44, (2011) 113

[35] F. T. Barcroft: Wear, 3, (1960) 440

[36] D. Philippon, M.I. De Barros Bouchet, Th. Le Mogne, E. Gresser, J.M. Martin, Trib.-Mat., Surf. and Interf., 1, (2007) 113

[37] D. Philippon, M.I. De Barros-Bouchet, Th. Le-Mogne, B. Vacher, O. Lerasle, J-M. Martin, Thin Solid Films 524, (2012) 191

[38] A.Heather et al. Tribology Letters 62:1 (2016)

[39] A. Heather et al. Journal of physical chemistry C 119:13 (2015)

[40] M. Filippo, A. Rossi,N. D. Spencer, Tribology Letters 35:1 (2009)

[41] N. N. Gosvami et al., Science 348 :6230 (2015) 102-106

[42] M. Crobu, Maura et al.Analytical and bioanalytical chemistry, 403:5 (2012) 1415-1432.

[43] Giannozi P. et al., J. Phys : Condens. Matter 21, (2009) 395502

[44] Perdew J.P, Burke K, Ernzerhof M, Phys. Rev. Letter. 77, (1996) 3865

[45] Monkhorst H. J, Pack J. D, Phys. Review B 13, (1976) 5188

[46] G. Zilibotti and M. C. Righi, Langmuir 27, (2011) 6862

[47] M. I. De Barros-Bouchet, J-M. Martin, D. Philippon, C. Matta, T. Le Mogne, M. Boehm, Japanese Society of Tribologists, Tribology Online 10, 1 (2015) 29

[48] Michelle J.S. Spencer, Andrew Hung, Ian K. Snook, Irene Yarovsky, Surf. Sci. 540, (2003) 420

[49] Shih H. D, Jona F, Jepsen D. W, Marcus P. M, Phys. Rev. Letter 46, (1981) 731

[50] Konicek A.R, Grierson D.S, Gilbert P.U.P.A, Sawyer W. G, Sumant A.V, Carpick R. W, Phys. Rev. Lett. 100, (2008) 235502

[51] M. I. De Barros-Bouchet, G. Zilibotti, C. Matta, M. C. Righi, L. Vandenbulcke, B. Vacher, J. M. Martin, J. Phys. Chem. C 116, (2012) 6966

[52] G. Zilibotti, S. Corni, M. C. Righi, Phys. Rev. Letters 111, (2013) 146101

[53] D. Philippon, M.I. De Barros Bouchet, T. Le-Mogne, O. Lerasle, J.M. Martin, Tribology International 44, (2009) 684

[54] Banfield et al., J. Org. Chem. 72, (2007) 4989

[55] A. G. Akhmadullina, et al., 29 (1993) 108

[56] Komvopoulos K, Do V, Yamaguchi E. S, Ryason P. R, Trib. Transaction 46 (2003) 315 
